\section{Positive induction at different sections of the performance chain*}

\author{
RICHARD V. KRANE and JAMES R. ISON† \\ University of Rochester, Rochester, N.Y. 14627
}

Rats received 95 differential conditioning trials followed by 30 massed extinction trials to the former $\mathrm{S}+$ either in succession (control procedure) or with each $\mathrm{S}+$ preceded by a trial to $S-$ (induction procedure). In Experiment 1, detention time on nonrewarded trials was $15 \mathrm{sec}$; in Experiment 2 it was $3 \mathrm{sec}$. The induction procedure provided generally faster response speeds and fewer avoidance reactions in extinction, these effects being most marked in the initial sections of the runway. Comparisons of the two experiments indicated that the animals ran faster and discriminated more quickly in Experiment 2 and that the facilitation effect of the induction procedure was more apparent in Experiment 1.

Extinction positive induction $(\mathrm{PI})$ is the greater resistance to extinction of the response to $\mathrm{S}+$ following discrimination learning if nonreinforced presentations of St are alternated with nonreinforced presentations of $\mathrm{S}-$, compared to a control group receiving the former $\mathrm{S}+$ alone (Senf \& Miller, 1967). In prior experiments on this phenomenon (Ison \& Krane, 1969; Krane \& Ison, 1970), we noted that extinction PI appeared to be strongest in the early sections of the performance chain to the former $\mathrm{S}+$ and unsystematically noticed an apparent reduction in avoidance reactions to $\mathrm{S}+$ with the induction procedure. The purpose of the two experiments reported here was to examine the effects of extinction PI on response speeds to the former St and, additionally, to assess the effect of this procedure on avoidance responses. Two separate experiments were run, which differed only in the duration of detention time in the unbaited goalbox on nonrewarded trials. The purpose of the second experiment, run with a very short detention time, was to see if the effects obtained in the first experiment could be retrieved under conditions that reduce the aversiveness of nonreward and lead, thereby, to generally enhanced resistance to extinction.

\section{METHOD \\ Apparatus}

Differential conditioning was conducted in a pair of parallel alleys, one channel white and one black (each $80 \times 3 \frac{1}{2} \times 4$ in.), to each of which a single gray startbox $\left(12 \times 3 \frac{1}{2} \times 4\right.$ in.) could be fixed. Three pairs of photocells connected to four Hunter clocks permitted start (start door to $6 \mathrm{in}$. from door), run (beginning 33 in.

*This research was supported by a National Science Foundation research grant, GB 14814. and by a United States Public Health Service training grant, MH 10825 .

t Recjuests for reprints should be sent to James R. Ison, Departinent of Psychology. University of Rochester, Rochester, N.Y. 14627. from the start door), and goal (beginning 72 in. from the start door) speeds to be measured over discrete 6-in. units, and provided a total (78-in.) speed measure. An aluminum food tray, placed behind a baffle at the end of the S+ alley, contained 10 37-mg Noyes pellets during training and was removed during extinction; no food tray was present in $\mathrm{S}$-.

\section{Subjects and Procedure}

The $S$ s were 24 naive male albino rats obtained from Holtzman Co. at about 90 days of age. Upon arrival, Ss were placed on a 23-h food-deprivation schedule (water ad lib) and were handled individually for about 5 min on each day of the 7-day pretraining period. During both pretraining and the experiments proper, Ss were allowed access to food for $1 \mathrm{~h}$ each day following handling or the experimental manipulations.

The day before discrimination training was begun, each $\mathrm{S}$ was given two rewarded goal placements in the St alley and two nonrewarded placements in $\mathrm{S}-$. During acquisition, Ss received 10 trials on Day 1 and 20 trials on Day 2, half to each stimulus, presented in Gellerman sequences. Days 3 and 4 involved a stimulus-generalization decrement-control procedure in which half of the Ss received 15 consecutive trials to $\mathrm{S}+$ alone (control condition) or $15 \mathrm{~S}$ - trials alternated with $15 \mathrm{~S}+$ trials (induction condition), counterbalanced over the 2 days. (This procedure insured that each $S$ had had experience with the sequence of stimulus presentations that would prevail in either the control or the induction condition in extinction.) The intertrial interval between successive $\mathrm{S}+$ trials was 1 min under both conditions. On Day 5 all Ss received $10 \mathrm{~S}+$ and $10 \mathrm{~S}$ - trials in a Gellerman sequence. These procedures resulted in 55 trials to $\mathrm{S}+$ and 40 trials to $S$ - in acquisition.

In extinction, Ss were assigned randomly to either the induction condition, in which case they received $15 \mathrm{~S}$ - trials alternated with 15 nonreinforced $S+$ trials on each of 2 days, or to the control condition, wherein they received only the 15 nonreinforced $S+$ trials on each day. Again, the 1-min intertrial interval between successive S+ trials was maintained for induction and control Ss alike.

The experimental procedures were identical for the $12 \mathrm{Ss}$ each in Experiments 1 and 2, except for the variable of goal-detention time. During acquisition, Ss in Experiment 1 were removed from the goal segment after $15-\mathrm{sec}$ detention but not before they had consumed the 10-pellet reward on S+ trials; in Experiment 2, Ss were removed from the goal segment after a 2- to 3-sec detention time on S- trials and immediately following consumption of the 10-pellet reward on St trials. During extinction, goal-detention time in both the nonrewarded $S+$ and $S-$ alleys was $15 \mathrm{sec}$ in Experiment 1 and $2.3 \mathrm{sec}$ in Experiment 2. In both experiments, Ss were removed only from the goal segments of the alley.

In addition to the speed measures,

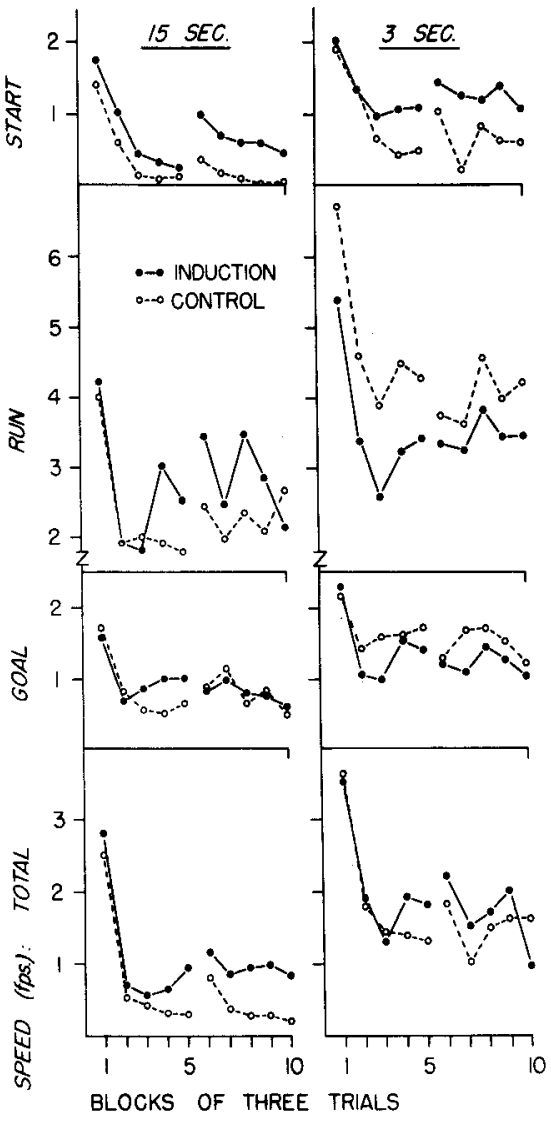

Fig. 1. Median response speeds in extinction for control and induction procedures of Experiment 1 (on the left) and Experiment 2 (on the right). 


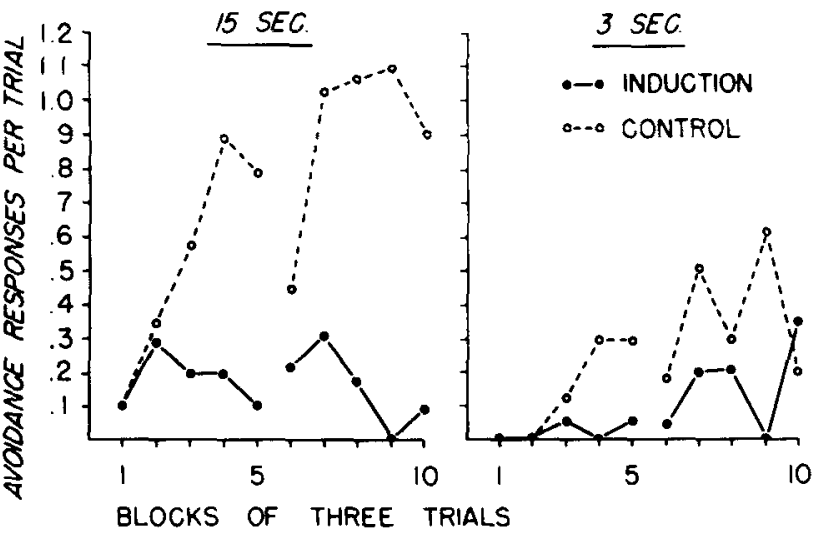

Fig. 2. Mean numbers of avoidance responses for control and induction procedures of Experiment 1 (on the left) and Experiment 2 (on the right).

record was made of frequency of avoidance behavior in extinction, defined as a turn 180 deg away from the goal.

\section{RESULTS AND DISCUSSION}

Experiment 1

Median speeds in blocks of three trials on each of the four speed measures are presented on the left side of Fig. 1. In general, the induction group, which received alternated nonreinforced trials to the former $S_{+}$and $S-$, responded with higher speeds in each measure. This difference was most consistent on the start measure and least so on the goal measure where on the second day the speeds overlapped to a considerable degree. Mann-Whitney U tests of speeds summed over all extinction trials revealed that the groups were significantly different on the start and total measures $(U=6, p<.03$, and $U=3, p<.01$, one-tailed) but not on the run and goal measures $(\mathrm{p}=.16$, $\mathrm{p}=.29$ ). Figure 2 depicts the mean number of avoidance responses per trial in blocks of three trials, the data for Experiment 1 on the left. It is clear that the induction procedure led to fewer avoidance reactions to the former $S+$ than did the control procedure; there was no overlap between the two groups in the numbers of avoidance responses summed over all 30 extinction trials $(U=0, p<.001)$, and, further, the control procedure led to more avoidance responses to the former $S+$ than did the induction procedure on responses to the former $\mathrm{S}-$; again, there was no overlap between these distributions $(U=0$, $\mathrm{p}<.001)$. It is interesting that avoidance reactions occur primarily in the initial portions of the runway. Of the 162 total avoidance reactions to the former $S+$ in control and induction procedures combined, 119 occurred in the first half of the runway and 79 occurred before the animals had entered 6 in. into the alley. Experiment 2

Comparable data for Experiment 2 are presented on the right sides of Figs. 1 and 2. On speed scores it appeared that the nonrewarded trials led to small induction effect on start and total measures but a reversal in the relative standings of the two groups on the run and goal measures. In fact, none of these differences in speed achieved traditional levels of significance; the usual induction effect on start speeds yielded $U=8, p=.066$ and on total speeds $\mathrm{U}=14, \mathrm{p}=.292$. The direction of differences on run and goal speeds was contrary to expectation: these yielded $\mathrm{U}=7, \mathrm{p}=.094$ and $\mathrm{U}=9, \quad \mathrm{p}=.18$ (two-tailed). Thus, although the pattern of relative differences resulting from the induction and control procedures was similar to that of Experiment 1, the speed data provide minimal evidence for an induction effect with these conditions of short detention time. Differences in the total number of avoidance reactions yielded more substantial evidence for induction. The induction procedure produced fewer such reactions to the former $\mathrm{St}$ than did the control procedure $(\mathrm{U}=5.5, \mathrm{p}<.032)$, and on the second day of extinction fewer avoidance reactions to the former $\mathrm{S}$ - than did the control procedure to $S+$ on that day $(U=2$, $p=.004)$. Of the 59 total responses to the former $\mathrm{S}+$ in both procedures combined, 38 occurred in the first half of the runway and 21 occurred before the rat had traversed its first 6 in.

\section{Comparisons of}

Experiments 1 and 2

Direct comparisons of the two experiments must be made with caution because they were conducted at different times, but the uniformity of Es, S population, and general experimental procedures indicates that these comparisons may be of some value. The most striking difference between the two experiments was that, as anticipated, the shift to a minimal detention time on animals run with the $3-\mathrm{sec}$ detention time ran much faster than those with the $15-\mathrm{sec}$ detention time. The distributions of scores were highly disparate. as only two animals in Experiment 1 entered the distribution of the animals run in Experiment 2. Except for start speeds in the control group $(\mathrm{U}=6, \mathrm{p}=.032)$, analyses of all four measures between the comparable groups yielded highly significant values $(0 \leqslant U \leqslant 3, .001 \leqslant p \leqslant .008)$. There was no overlap in the distribution of avoidance responses between the control groups, with the control groups in Experiment 1 averaging 21.8 responses and in Experiment 2 averaging but $7.5 \quad(\mathrm{U}=0$, $p=.001)$. The difference in the two induction groups was in the same direction (5.5 vs 2.7 ), but there was considerable overlap between the two distributions $(\mathrm{U}=10, \mathrm{p}=.12)$. These large differences in extinction performance between the two experiments were paralleled by similar differences in speeds to $S$ on terminal acquisition performance (start, $U=27$, $\mathrm{p}<.01$, run, $\mathrm{U}=12, \mathrm{p}<.001 ;$ total, $\mathrm{U}=6.5, \mathrm{p}<.001$; goal speeds were identical), although such terminal differences were not generally present on $\mathrm{S}+$ speeds (except on the run measure, $\mathrm{U}=23, \mathrm{p}<.01)$. On the first 30 acquisition trials, the longer detention times on $\mathrm{S}$ - generally depressed speeds on $\mathrm{S}+$ (run, $\mathrm{U}=22, \mathrm{p}<.01 ;$ goal, $\mathrm{U}=20$, $p<.001$; total, $U=11, p<.001)$ but had little effect on speeds to $\mathrm{S}$-. This anomalous effect resulted because animals in the short detention group learned to inhibit responses to $S$-in fewer trials than did animals in the long detention group.

It had been anticipated that the shorter detention time following nonreward in Experiment 2 would lead to the slower extinction of the (generalized) approach to S- and, thus, a reduced rate of differentiation. This tumed out not to be the case. The trials in the first 2 days were arranged as 15 pairs, one $S+$ and one $S$, and these pairs ordered according to whether $\mathrm{S}+$ or $\mathrm{S}$ - was the greater. In terms of the mean number of positive pairs $(\mathrm{S}+>\mathrm{S}-)$, animals in Experiment 2 averaged 10.75, those in Experient 1 averaged 8.83 . In terms of number of pairs to a criterion of three consecutivepositive pairs, animals in Experiment 2 averaged 5.92, those in Experiment 1 averaged 8.25. Analyses indicated that the distributions associated with these statistics were reliably disparate $(\mathrm{U}=22.5, \mathrm{U}=24$, $\mathrm{p}<.02$, two-tailed). These analy ses suggest that the short detention time led to faster differentiation of performances associated with $\mathrm{S}+$ and $\mathrm{S}-$. This is a surprising finding, for it is generally found that variables that enhance or reduce resistance 
to extinction conversely reduce or enhance rate of differentiation by appropriately varying the number of trials necessary before speeds to $\mathrm{S}$ - diverge from the speeds to $\mathrm{S}+$. Sometimes it has been reported that a variable which affects extinction performance does not affect rate of differentiation, which results because the effect of the variable on speeds to $S-$ is paralleled by its effect on speeds to S+ (for example, Spence, Goodrich, \& Ross, 1959; Experiment 1). A similar effect was noted in the early trials of the present set of experiments in which long-duration confinement produced a generalized decrement in speeds on $\mathrm{S}+$ as well as $\mathrm{S}-$-; by itself, however, this generalized effect should have resulted in equal rates of differentiation in the two groups rather than faster learning in the short detention group. But this may be the time to exercise the caveat expressed in the introduction to this section and to withhold judgment on the effect of detention time on rate of differentiation until it has been manipulated in the context of a single experiment.

The similarities between the two experiments are several; although the positive induction effect may be more pronounced under conditions of long detention time, the facilitory effects of the interposed $\mathrm{S}-$ trials are evidently most prominent in the initial sections of the runway with either procedure. Both experiments indicated that the induction procedure markedly reduced the number of avoidance responses that occur in extinction, and both agreed in placing the locus of these avoidance responses predominantly in the first sections of the alley, with about $50 \%$ of these responses occurring immediately on the presentation of the nonreinforced stimulus. It may be that the major effect of the induction procedure is to reduce the occurrence of avoidance responses on the former $\mathrm{S}+$, and thus, because avoidance responses are otherwise most likely to occur on the initial presentation of that stimulus, the PI effect is most pronounced at the beginning of the runway.

\section{REFERENCES}

ISON, J. R., \& KRANE, R. V. Induction in differential instrumental conditioning. Journal of Experimental Psychology, 1969, 80, 183-185.

KRANE, R. V., \& ISON, J. R. Positive induction in differential instrumental conditioning: The effect of extended acquisition training. Journal of Comparative \& Physiological Psychology, 1970, in press.

SENF, G. M., \& MILLER, N. E. Evidence for Pavlovian induction in discrimination learning. Journal of Comparative \& Physiolosical Psychology, 1967,64, 121-127.

SPENCE. K. W., GOODRICH, K. P., \& ROSS. L. E. Performance in differential conditioning

and discrimination learning as a function of hunger and relative response frepuency.

Journal of Experimental Psychology, 1959, $58,8-16$.

\title{
Peeping in chicks as a function of environmental change
}

\author{
CLARE FULLERTON, JULIA C. BERRYMAN, and W. SLUCKIN \\ University of Leicester, Leicester, England
}

Peeping was measured in 1-day-old and 4-day-old chicks in four different testing conditions. No differences were found between the two ages, but peeping increased markedly with the degree of environmental change. This latter variable may have been overlooked in past work because of the prevailing interest in changes of behavior as a function of disruption of social imprinting bonds.

Vocalizations of the domestic chick range from "pleasure calls" or twitters to "distress calls" or peeps, which in the most extreme form may be described as shrieks (Andrew, 1964). It is well known that peeps are readily evoked by a wide range of stimuli, occurring when birds are hungry, in pain, under restraint, or when approached by a large object (Collias, 1952). Peeping also occurs when a chick is separated from its mother or companions (Bermant, 1963; McBride et al, 1969); and it is a response to ambient temperatures below or above $88^{\circ}-90^{\circ} \mathrm{F}$ (Kaufman \& Hinde, 1961; Herbert \& Sluckin, 1969). The question we wished to ask was to what extent certain disturbances of the physical environment other than those mentioned above could affect peeping.

\section{PRELIMINARY EXPERIMENT} Apparatus

The only pieces of apparatus used were standard grey cardboard chick rearing boxes, $1 \mathrm{ft}$ square. Constant heating and lighting were provided by $60-\mathrm{W}$ light bulbs placed centrally, which gave a temperature of $85^{\circ} \mathrm{F}$ directly underneath and $75^{\circ} \mathrm{F}$ around the sides of the box. Food and water were always available.

\section{Subjects}

The Ss were 80 chicks hatched from Cobb eggs in the laboratory. Chicks were allowed to dry off in the incubator before being isolated in the rearing boxes, where they remained until testing.

\section{Procedure}

The chicks were placed at hatching in groups of four in the chick rearing boxes. Testing took place at either 1 or 4 days of age. Two tests were given to each age group, and the chicks were thus allocated at random to one of four possible groups. Peeps were recorded from a single chick in a 5-min test following the removal of the chick from its rearing box to another box of exactly the same design but containing

*We should like to thank Mr. P. Kerrigan for having helped with some of the testing. no other chicks. In the second experimental condition, peeps were recorded from a single chick in a 5-min test following the removal of its three penmates from the rearing box. In both cases the chicks were tested in isolation, but in one case the environment was familiar and in the other it was strange, although similarly designed. It should be noted that the chicks removed to another similar box were also handled by the $\mathrm{E}$, whereas those left in the rearing box were not.

Results

All Ss were highly distressed in the test period (see Table 1), and the differences between the groups were not significant.

\section{Discussion}

It looks as though when there is a high level of "distress," as at social bond disruption, differences in the amount of handling and in the physical environment do not have enough impact to be reflected in a rise in peeping. Our main experiment, however, in which all the chicks were singly reared, was an attempt to find out whether, when there are no social bonds, relatively slight degrees of "stimulus contrast"-a term used by Andrew (1964)-can bring about differences in peeping.

\section{MAIN EXPERIMENT Apparatus}

Chicks were reared and tested in three of our four experimental conditions in boxes made of grey cardboard measuring $1 \times 1 \times 1 \mathrm{ft}$. Heat and light were provided by a centrally placed $60-\mathrm{W}$ bulb which gave a temperature of $85^{\circ} \mathrm{F}$ directly underneath and $75^{\circ} \mathrm{F}$ around the sides of the box. Food and water were always available.

An open field was used in the fourth experimental condition. This was built of white hardboard and measured $2 \times 2 \times 1 \mathrm{ft}$ high. One wall was made of white gauze to allow the $\mathrm{E}$ to observe the chicks unnoticed by them. The open field was lit by a $60 . \mathrm{W}$ bulb placed above it and to one side. The temperature was maintained at approximately $76^{\circ} \mathrm{F}$ 